\title{
Bullying entre Estudantes com e sem Características de Dotação e Talento
}

\author{
Bullying among Students with and without Characteristics of Giftedness
}

\author{
Juliana Célia Oliveira* \& Altemir José Gonçalves Barbosa \\ Universidade Federal de Juiz de Fora, Juiz de Fora, Brasil
}

\begin{abstract}
Resumo
Para comparar a ocorrência de bullying entre alunos com e sem características de dotação e talento (D\&T), aplicou-se um questionário em 339 estudantes do ensino fundamental, sendo que 59 deles possuíam D\&T. Não foram identificadas diferenças significativas entre os subgrupos com e sem D\&T quanto ao envolvimento com bullying, aos tipos de agressões sofridas e a variáveis sociométricas. Os alunos com D\&T adotam mais frequentemente o comportamento de pedir auxílio para combater o bullying. Os discentes com talento para arte são mais vitimizados que os colegas com D\&T em outros domínios. Ainda que poucas variáveis relacionadas ao ajustamento socioemocional tenham sido investigadas, os resultados permitem inferir que estudantes com D\&T não diferem necessariamente dos pares nesse âmbito do desenvolvimento humano.

Palavras-chave: Violência na escola, superdotação, desenvolvimento socioemocional.
\end{abstract}

\begin{abstract}
To compare the occurrence of bullying among students with and without characteristics of giftedness and talent it was applied a questionnaire to 339 elementary school students, being 59 of them gifted students. No significant differences were found between subgroups with and without giftedness in regard to their involvement with bullying, types of aggression suffered and sociometric variables. Gifted students more often adopt the behavior of calling for help to combat bullying. Students with talent for arts tend to be more victimized than the ones gifted in other domains. Although few variables related to socioemotional adjustment have been investigated, the results may imply that students with giftedness do not necessarily differ from peers regarding that aspect of human development.

Keywords: Violence in school, giftedness, socioemotional development.
\end{abstract}

Nas últimas duas décadas, a violência escolar tem sido alvo de preocupação crescente, tornando-se um problema que transcende os muros escolares. Uma das manifestações de violência na escola, que tem recebido atenção significativa pela literatura especializada, é o fenômeno denominado bullying (Centro de Empreendedorismo Social e Administração em Terceiro Setor [CEATS], 2010; Malta et al., 2010; B. O. Pereira, 2008; Smith, 2002). Os primeiros estudos sobre o tema foram publicados por Olweus (1993), na Noruega, e, desde então, o interesse pela temática aumentou, passando a ser considerado problema de saúde pública (Almeida, Silva, \& Campos, 2008; Fante, 2005; Lopes Neto, 2005; Nansel, Craig, Overpeck, Saluja, \& Ruan, 2004). Consequentemente, ganhou destaque em diversos países, ampliando expressivamente a produção científica e as políticas públicas que visam combatê-lo e/ou preveni-lo (Oliveira, 2007).
Bullying é uma palavra de origem inglesa e não possui tradução exata para o português. Esse tipo de violência tem sido definido como um subconjunto de comportamentos agressivos, caracterizado por sua natureza intencional e constante e marcado pela assimetria de poder entre os envolvidos (Botelho \& Souza, 2007; Fante, 2005; Lopes Neto, 2005; Oliveira \& Votre, 2006; Pereira, 2008; Piedra, Lago, \& Massad, 2006; Seixas, 2005).

Os atos de agressividade que envolvem o fenômeno bullying podem ser manifestados por: agressões físicas (bater, empurrar, dar pontapés, etc.), comportamentos verbais (caçoar, chamar por nomes ofensivos, etc.), comportamentos de manipulação social ou indiretos (excluir, ignorar, espalhar rumores, etc.), comportamentos de maus-tratos psicológicos (ameaçar, fazer gestos ou expressões faciais provocadores e/ou ameaçadores, etc.) e ataques à propriedade (furto, extorsão, destruição deliberada de materiais/objetos etc.; Lisboa, Braga, \& Ebert, 2009; Seixas, 2005; Smith, 2002).

Quanto aos envolvidos com bullying, observa-se a diferenciação dos seguintes papéis: vítimas passivas;

•E-mail: julianaoliveirapsi@gmail.com 
vítimas agressivas; observadores; ou agressores (Fante, 2005; Lopes Neto, 2005; Seixas, 2005; Smith, 2002). De acordo com os autores, as vítimas passivas podem ser caracterizadas como inseguras, ansiosas e incapazes de se defenderem. As vítimas agressivas geralmente apresentam temperamento exaltado, retaliam o ataque e/ou agridem a outros. Os observadores ou não participantes são aqueles que convivem com a violência, mas não se manifestam sobre ela. Os agressores, individualmente ou em grupo, podem ser os líderes ou seguidores dessa forma de agressão.

Nogueira (2005), por sua vez, define as vítimas como alunos frágeis, que se sentem desiguais ou prejudicados e que dificilmente pedem ajuda. Além disso, os alunos vitimizados podem apresentar poucas competências sociais, sendo pouco assertivos ou respondendo de forma provocativa (B. O. Pereira, 2008). Já os agressores são definidos por Lopes Neto (2005) como tipicamente populares, impulsivos, envolvidos em uma variedade de comportamentos antissociais, geralmente mais fortes que os pares vitimizados, podendo sentir prazer em dominar, controlar e causar danos a outros.

Ressalta-se que o bullying tem maiores chances de ocorrer entre crianças e adolescentes com necessidades educacionais especiais (Carter \& Spencer, 2006; Flynt \& Morton, 2007; B. O. Pereira, 2008; Smith, 2002), pois parcela significativa desse grupo de pessoas é composta por indivíduos com menor repertório de competências sociais e com poucas relações de amizade, tendo maiores chances de serem rejeitados. Há que se ressaltar, contudo, que se trata de um grupo heterogêneo, abrangendo indivíduos com deficiências (deficiência mental etc.), com problemas menos severos (dificuldades de aprendizagem específicas etc.) e com altas capacidades (dotação e talento; Ministério da Educação, 2008).

O último subgrupo de necessidades educacionais especiais é denominado pelo Ministério da Educação do Brasil (2008) como estudantes com altas habilidades/ superdotação. Trata-se de alunos que demonstram potencial elevado nos domínios: liderança, psicomotricidade, criativo-produtivo, intelectual, acadêmico e artes, exibidos isolados ou combinadamente. Acrescentam-se ainda, como características, elevada criatividade e grande envolvimento na aprendizagem e na realização de tarefas em áreas de seu interesse (Secretaria de Educação Especial, 1995).

É preciso destacar que outros termos também têm sido utilizados para descrever essa condição, tais como dotação e talento. Güenther (2006) se posiciona contra o termo superdotação, uma vez que, além de possuir equívocos na tradução conceitual, denota pouco respeito e antipatia. No lugar dele, propõe que existam áreas e domínios de capacidade. Para a autora, talento é o que melhor designa esse tipo de necessidade educacional especial, pois se refere muito mais à aquisição do que à herança de determinada capacidade (Barbosa et al., 2008).
Mais do que uma pluralidade terminológica e/ou problemas de tradução de termos utilizados para caracterizar indivíduos com determinado talento, dotação ou capacidade superior, essa diversidade representa diferenças epistemológicas e conceituais profundas que não serão discutidas no escopo do presente trabalho. Todavia, destaca-se que a opção por usar a expressão dotação e talento (D\&T), e não os termos superdotação, altas habilidades, superdotado etc., é decorrente, dentre outros, dos motivos destacados por Güenther (2006). Ressalta-se, ademais, a preferência por não se utilizar os termos 'dotado' e/ou 'talentoso', já que eles podem sugerir que se trata de uma condição estável e, até mesmo, imutável. Se usada em conjunto, a expressão dotação e talento (D\&T) tende a denotar uma condição relacionada ao desenvolvimento humano (Horowitz, 2009), bem como indica tratar-se de uma característica humana decorrente da interação entre organismo e ambiente (Kaufman \& Sternberg, 2008). Dessa forma, as pessoas desenvolvem, em determinado contexto social e histórico, essas características ou comportamentos, não sendo, assim, dotada (superdotada) e talentosa.

No processo de identificação e desenvolvimento de estudantes com D\&T, observa-se uma ênfase no atendimento às necessidades cognitivas e acadêmicas. Entretanto, pouca atenção tem sido dada ao desenvolvimento socioemocional desses alunos (Alencar \& Fleith, 2001). Alguns mitos difundidos, as poucas pesquisas existentes e as publicações com resultados discrepantes e controversos a respeito do ajustamento socioemocional dos indivíduos com D\&T demonstram a necessidade de mais investigações sobre o assunto (Alencar, 2007). No caso desses alunos, um desenvolvimento cognitivo avançado não implica necessariamente em um desenvolvimento afetivo maduro (Ourofino \& Guimarães, 2007; Webb, 1993). Os autores mencionam algumas características afetivas desse grupo de crianças, como dificuldade nos relacionamentos sociais, dificuldade em aceitar críticas, intensidade de emoções, perfeccionismo etc.

Alguns pesquisadores sugerem que principalmente jovens com D\&T intelectual extremos possuem maior predisposição a problemas socioemocionais (Alencar \& Virgolim, 2001; Peterson, 2009), além de apresentarem maiores riscos para a depressão, ansiedade e suicídio (Peterson, 2001). No entanto, outros estudos enfatizam que os alunos identificados com D\&T acadêmico tendem a apresentar níveis mais altos do funcionamento social e comportamentos pró-sociais, se comparados aos pares não identificados (Estell et al., 2009; Lehman \& Erdwins, 2004).

Güenther (2006) sinaliza que, frequentemente, o indivíduo com D\&T é percebido como 'diferente' dos outros, principalmente no convívio escolar, o que pode acarretar implicações negativas para várias áreas de relacionamento humano. Dependendo do meio social na escola, muitos estudantes com D\&T são estereotipados 
e evitar ameaças a tal condição torna-se uma prioridade para eles (Cross, 2001). Assim, de acordo com o autor, enquanto alguns alunos podem atuar de forma violenta, outros parecem interiorizar o sentimento de raiva, o que dificulta a criação de estratégias de enfrentamento social.

O bullying, como um dos problemas mais graves nos relacionamentos entre pares, afeta negativamente todos os envolvidos. No entanto, como afirmam Peterson e Ray (2006a) e Schuler (2002), os alunos com D\&T se diferem dos outros de forma significativa. Para os autores, características como serem emocionalmente intensos, sensíveis e destacados por suas próprias expectativas e habilidades podem torná-los mais vulneráveis. Um estudo sobre prevalência e efeitos do bullying, realizado com 432 alunos com D\&T, pertencentes à educação infantil e ao ensino fundamental, em diferentes escolas nos Estados Unidos, revelou que $67 \%$ dos participantes tinham experimentado pelo menos um dos 13 tipos de bullying listados na pesquisa (Peterson \& Ray, 2006a). No último ano do ensino fundamental, houve um número maior de estudantes envolvidos em intimidação; $16 \%$ eram agressores e $29 \%$ tinham pensamentos violentos. Em outra parte do estudo (Peterson \& Ray, 2006b), os autores procuraram investigar, através de pesquisa qualitativa, o impacto emocional causado pelo envolvimento com o bullying em 57 estudantes. No entanto, como ambos os estudos não compararam jovens com $\mathrm{D} \& \mathrm{~T}$ com os pares que não possuíam essa condição, pode-se apenas especular que os participantes possuíam alta sensibilidade às provocações.

Diferentemente, no estudo de Estell et al. (2009), com 484 estudantes (sendo que 74 eram alunos com D\&T e 41 possuíam deficiências leves), foi observado que tanto os professores quanto os alunos percebiam os estudantes com deficiências leves como mais envolvidos em bullying como agressores. Já os alunos com D\&T eram percebidos como menos propensos a estarem envolvidos com o fenômeno. Esses resultados parecem ser coerentes com outras pesquisas que afirmam que estudantes com D\&T tendem a apresentar maiores estratégias de enfrentamentos e resolução de problemas sociais (Preuss \& Dubow, 2004), maiores níveis de empatia e de raciocínios pró-sociais (Hay, 2008) e altos níveis de aceitação dos colegas (Luftig \& Nichols, 1990), se comparados aos pares sem características de D\&T.

Em outro sentido, Parker (2010) buscou determinar se as taxas de bullying e vitimização diferiam entre alunos com D\&T e alunos que apresentam alto desempenho, mas que não eram identificados. Na pesquisa, não foram encontradas diferenças significativas entre os dois grupos no que se refere ao envolvimento com essas práticas de agressão.

Apesar do rápido crescimento de pesquisas sobre bullying observado internacionalmente (Peterson \& Ray, 2006a) - o estado da arte não é muito diferente no caso de D\&T - há, ainda, poucos estudos sobre bullying entre estudantes com D\&T. No Brasil, a produção científica nos dois campos, ou seja, bullying e D\&T, é muito res- trita e praticamente inexistente quando associa ambos os temas. Chagas (2008) apenas discute a necessidade de tais pesquisas, ressaltando em sua tese que, se forem consideradas as habilidades intra e interpessoais e os aspectos afetivos e sociais dos 42 adolescentes participantes de sua pesquisa, $1 / 3$ deles com talento artístico e mais de $40 \%$ com talento acadêmico informaram envolvimento em brigas. Aproximadamente a metade dos adolescentes da pesquisa recebeu algum apelido pejorativo e cerca de $20 \%$ dos adolescentes com talento artístico e acadêmico sofreram algum tipo de agressão verbal ou física, sendo que todos os adolescentes com duplo talento informaram terem sido agredidos fisicamente.

A controvérsia existente quanto ao desenvolvimento social e afetivo de pessoas com D\&T e ao seu envolvimento ou não com bullying não deve representar um impedimento para a realização de pesquisas sobre esse tema. Ao contrário, deve servir como estímulo, pois estudar as características de tais pessoas pode tanto revelar vulnerabilidades desse grupo quanto identificar fontes de resiliência.

$\mathrm{O}$ presente estudo teve como objetivo investigar a ocorrência de bullying entre alunos com e sem características de D\&T. Especificamente, além de caracterizar os participantes, os subgrupos com e sem D\&T foram comparados quanto ao tipo de envolvimento com bullying escolar, às formas de agressão utilizadas, às reações perante essa forma de violência e, ainda, a variáveis sociométricas (número de amigos e ser mencionado como amigo).

\section{Método}

\section{Participantes}

Fizeram parte do estudo 339 alunos pertencentes às turmas de $6^{\circ}$ ao $9^{\circ}$ ano do ensino fundamental de uma escola pública da zona da mata mineira, sendo que $43,7 \%$ deles $(n=148)$ eram do sexo masculino. Do total de participantes, $59(17,4 \%)$ estudantes foram identificados previamente com características de D\&T, sendo que 25 $(42,4 \%)$ destes eram do sexo masculino.

Os participantes com D\&T eram vinculados a um programa que tem como objetivo identificar e desenvolver estudantes com essas características. O processo de identificação utiliza múltiplas fontes de informação, tais como testes de inteligências, nomeação por professores e nomeação por pais. Uma descrição detalhada dos instrumentos e procedimentos usados pelo programa pode ser encontrada em Pereira (2010).

\section{Instrumentos}

Para coleta de dados, foi utilizado o Questionário de Bullying (Barbosa, 2009). Trata-se de uma medida adaptada para o contexto brasileiro do questionário que Pereira (2008) desenvolveu em Portugal. Essa autora, por sua vez, teve como base o instrumento bullying de Dan Olweus, construído em 1989. A versão brasileira do questionário contém 42 questões divididas em quatro 
sessões: (a) caracterização sócio-demográfica; (b) ser vítima de bullying; (c) ser agressor; e (d) ambiente escolar, na qual estão incluídas variáveis relacionadas à aceitação/rejeição pelos pares, à nomeação de colegas, aos recreios/intervalos e playgrounds.

\section{Procedimento}

Após aprovação pelo Comitê de Ética em Pesquisa (CAAE - 0192.0.180.00.09), o diretor da instituição e pais/responsáveis pelos alunos consentiram livre e esclarecidamente sobre a participação dos estudantes na pesquisa. Por se tratarem, em sua maioria, de adolescentes, além da autorização dos pais, os alunos também puderam expressar-se a respeito de sua participação ou não na pesquisa, pois de acordo com o Estatuto da Criança e do Adolescente (1990), os adolescentes têm direito à liberdade, ao respeito e à dignidade, podendo, como pessoas humanas em processo de desenvolvimento, se expressar e opinar livremente.

A coleta de dados foi efetuada no próprio colégio, em horário escolar, e realizada por um pesquisador, com a presença do professor em sala de aula. Não houve controle do tempo para a resposta do instrumento, contudo, a coleta se realizou em aproximadamente 40 minutos em cada turma.
Em decorrência das características do instrumento utilizado, a análise dos dados foi essencialmente quantitativa. Além de estatística descritiva, foram utilizadas provas inferenciais, adotando um nível de significância de 0,05 , uma vez que ele se mostrou adequado ao delineamento do estudo, ao instrumento de coleta de dados e ao tipo de amostragem.

A variável cor/raça foi analisada tendo como base o critério de classificação de cor/raça do Instituto Brasileiro de Geografia e Estatística (2003). O Critério Brasil de Classificação Econômica da Associação Brasileira de Empresas de Pesquisa (2010) foi adotado para inferir o nível econômico.

No caso do bullying, os participantes foram classificados como vítimas, agressores, vítimas agressivas e não envolvidos diretamente (Seixas, 2005). Foram consideradas vítimas os indivíduos que sofreram por três vezes ou mais qualquer tipo de agressão no trimestre anterior ao da coleta de dados. Considerou-se agressor o estudante que cometeu três ou mais agressões nesse mesmo intervalo. Já as vítimas agressivas foram consideradas como aquelas que apresentaram essas duas condições, isto é, sofrer e cometer três ou mais agressões durante três meses. Esses critérios têm sido adotados nos estudos de Pereira (2008), em Portugal, e também foram usados no Brasil para que as comparações interculturais fossem mais precisas.

Tabela 1

Caracterização Demográfica dos Participantes

\begin{tabular}{|c|c|c|c|c|}
\hline & & \multicolumn{2}{|c|}{$\begin{array}{c}\text { Aluno com característica } \\
\text { de dotação e talento }\end{array}$} & \multirow[t]{2}{*}{$\begin{array}{c}\text { Comparações } \\
\text { intergrupos }\end{array}$} \\
\hline & & Não & Sim & \\
\hline Sexo & $\begin{array}{l}\text { Masculino } \\
\text { Feminino }\end{array}$ & $\begin{array}{l}\mathrm{n}=123 ; 43,9 \% \\
\mathrm{n}=157 ; 56,1 \%\end{array}$ & $\begin{array}{l}\mathrm{n}=25 ; 42,4 \% \\
\mathrm{n}=34 ; 57,6 \%\end{array}$ & $\begin{array}{l}\chi_{0}^{2}=0,048 ; g l=1 \\
p>0,05\end{array}$ \\
\hline Idade & & $\begin{array}{l}M 12,79 \\
D P=1,273 \\
10>17\end{array}$ & $\begin{array}{l}M 11,98 \\
D P=1,091 \\
10>14\end{array}$ & $\begin{array}{l}U=5324,000 \\
p<0,001\end{array}$ \\
\hline Ano & $\begin{array}{l}6^{\circ} \text { Ano } \\
7^{\circ} \text { Ano } \\
8^{\circ} \text { Ano } \\
9^{\circ} \text { Ano }\end{array}$ & $\begin{array}{l}\mathrm{n}=57 ; 20,4 \% \\
\mathrm{n}=72 ; 25,7 \% \\
\mathrm{n}=74 ; 26,4 \% \\
\mathrm{n}=77 ; 27,5 \%\end{array}$ & $\begin{array}{l}\mathrm{n}=23 ; 39,0 \% \\
\mathrm{n}=15 ; 25,4 \% \\
\mathrm{n}=18 ; 30,5 \% \\
\mathrm{n}=3 ; 5,1 \%\end{array}$ & $\begin{array}{l}\chi_{0}^{2}=17,840 ; \mathrm{gl}=3 \\
\mathrm{p}<0,001\end{array}$ \\
\hline Cor/Raça & $\begin{array}{l}\text { Preto(a) } \\
\text { Branco(a) } \\
\text { Pardo(a) } \\
\text { Amarelo(a) } \\
\text { Indígena }\end{array}$ & $\begin{array}{l}\mathrm{n}=30 ; 10,9 \% \\
\mathrm{n}=173 ; 62,9 \% \\
\mathrm{n}=58 ; 21,1 \% \\
\mathrm{n}=6 ; 2,2 \% \\
\mathrm{n}=8 ; 2,9 \%\end{array}$ & $\begin{array}{l}\mathrm{n}=2 ; 3,4 \% \\
\mathrm{n}=41 ; 69,5 \% \\
\mathrm{n}=13 ; 22,0 \% \\
\mathrm{n}=2 ; 3,4 \% \\
\mathrm{n}=1 ; 1,7 \%\end{array}$ & $\begin{array}{l}\chi_{0}^{2}=3,777 ; g l=4 \\
p>0,05\end{array}$ \\
\hline Nível Econômico & $\begin{array}{l}\text { Classe B2 } \\
\text { Classe C1 } \\
\text { Classe C2 } \\
\text { Classe D } \\
\text { Classe E }\end{array}$ & $\begin{array}{l}\mathrm{n}=59 ; 21,1 \% \\
\mathrm{n}=101 ; 36,1 \% \\
\mathrm{n}=102 ; 36,4 \% \\
\mathrm{n}=17 ; 6,1 \% \\
\mathrm{n}=1 ; 0,3 \%\end{array}$ & $\begin{array}{l}\mathrm{n}=17 ; 28,8 \% \\
\mathrm{n}=22 ; 37,3 \% \\
\mathrm{n}=16 ; 27,1 \% \\
\mathrm{n}=4 ; 6,8 \% \\
\mathrm{n}=0 ; 0 \%\end{array}$ & Phi $=0,091 ; p>0,05$ \\
\hline
\end{tabular}


Oliveira, J. C. \& Barbosa, A. J. G. (2012). Bullying entre Estudantes Com e Sem Características de Dotação e Talento.

\section{Resultados}

Observou-se que não houve diferenças estatísticas entre os dois subgrupos investigados no que se refere à proporção de participantes do sexo masculino e feminino e às diferenças relacionadas à cor/raça e ao nível econômico (Tabela 1). No entanto, diferenças puderam ser percebidas no que se refere à idade e ao ano escolar, já que houve

Tabela 2

Envolvimento com o Bullying, Formas de Agressões e Variáveis Sociométricas dos Participantes

\begin{tabular}{|c|c|c|c|c|}
\hline & \multirow[t]{2}{*}{ Variáveis } & \multicolumn{2}{|c|}{$\begin{array}{l}\text { Aluno com característica } \\
\text { de dotação e talento }\end{array}$} & \multirow[t]{2}{*}{$\begin{array}{l}\text { Comparações } \\
\text { intergrupos }\end{array}$} \\
\hline & & Não & Sim & \\
\hline Envolvimento com Bullying & $\begin{array}{l}\text { Não envolvido } \\
\text { Vítima } \\
\text { Agressor } \\
\text { Vítima Agressiva }\end{array}$ & $\begin{array}{l}\mathrm{n}=198 ; 70,7 \% \\
\mathrm{n}=33 ; 11,8 \% \\
\mathrm{n}=26 ; 9,3 \% \\
\mathrm{n}=23 ; 8,2 \%\end{array}$ & $\begin{array}{l}\mathrm{n}=41 ; 69,5 \% \\
\mathrm{n}=10 ; 16,9 \% \\
\mathrm{n}=3 ; 5,1 \% \\
\mathrm{n}=5 ; 8,5 \%\end{array}$ & $\begin{array}{l}\chi_{0}^{2}=2,044 ; \mathrm{gl}=3 \\
\mathrm{p}>0,05\end{array}$ \\
\hline Tipo de Agressão & $\begin{array}{l}\text { Física } \\
\text { Não física } \\
\text { Ambos }\end{array}$ & $\begin{array}{l}\mathrm{n}=4 ; 7,3 \% \\
\mathrm{n}=42 ; 76,4 \% \\
\mathrm{n}=9 ; 16,4 \%\end{array}$ & $\begin{array}{l}\mathrm{n}=1 ; 6,7 \% \\
\mathrm{n}=11 ; 73,3 \% \\
\mathrm{n}=3 ; 20,0 \%\end{array}$ & $\begin{array}{l}\chi_{0}^{2}=0,111 ; g l=2 \\
p>0,05\end{array}$ \\
\hline Quantidade de amigos & & $\begin{array}{l}M=5,14 \\
D P=3,528\end{array}$ & $\begin{array}{l}M=5,33 \\
D P=3,328\end{array}$ & $\begin{array}{l}\mathrm{U}=7308,000 \\
\mathrm{p}>0,05\end{array}$ \\
\hline Citações como melhor amigo & & $\begin{array}{l}M=4,49 \\
D P=2,39\end{array}$ & $\begin{array}{l}M=4,63 ; 0 \\
D P=1,956\end{array}$ & $\begin{array}{l}\mathrm{U}=7653,000 \\
\mathrm{p}>0,05\end{array}$ \\
\hline
\end{tabular}

As respostas das vítimas quanto às formas como o bullying tem ocorrido foram classificadas em agressões dos tipos: físicas; não físicas; ou ambas (Tabela 2). As agressões físicas, menos frequentes, envolvem ataque direto ao corpo da vítima, como bater, dar socos, murros e/ou chutes, etc. Já as agressões não físicas, mais frequentes neste estudo, podem ser tanto diretas, como colocar apelidos ou nomes ofensivos e pegar objetos sem permissão, quanto indiretas, como falar escondido, parar de conversar, espalhar mensagens por telefone ou internet etc. Houve estudantes que relataram sofrer ambos os tipos de agressões. Observou-se que tanto os alunos com D\&T quanto os não identificados se assemelham no que se refere aos tipos de agressões sofridas.

Pode-se notar, também, que estudantes com e sem D\&T não diferiram em relação às variáveis sociométricas investigadas (Tabela 2). Desse modo, tanto a quantidade de amigos que têm na escola quanto o número de vezes que foram citados pelos pares como melhor amigo foram equivalentes para os subgrupos.

Ao analisar somente os alunos sem características de D\&T, foram encontradas associações significativas entre os tipos de envolvimento com bullying e as variáveis sexo e idade. Observou-se que o sexo masculino apresentou mais alunos identificados com D\&T nos $6^{\circ}$ e $7^{\circ}$ anos, sendo, portanto, mais novos que os colegas sem D\&T.

Constatou-se que 29,3\% $(\mathrm{n}=82)$ dos alunos sem características de D\&T e 30,5\% $(\mathrm{n}=18)$ dos estudantes com essa necessidade educacional especial estavam envolvidos diretamente com o bullying. Ao classificar esses estudantes como vítimas, agressores e vítimasagressivas (Tabela 2) e comparar os subgrupos, não foi encontrada diferença significante. 
ferenças entre os participantes com e sem D\&T em todas as reações propostas no instrumento: dizer a um professor ou diretor $(\mathrm{Phi}=-0,0124 ; \mathrm{p}>0,05)$; contar ao pai ou responsável $(\mathrm{Phi}=-0,068 ; \mathrm{p}>0,05)$; contar a um ou dois amigos (Phi $=-0,114 ; \mathrm{p}>0,05)$; não dizer a ninguém sobre as agressões que têm sofrido $(\mathrm{Phi}=0,099 ; \mathrm{p}>0,05)$; contar aos amigos $(\mathrm{Phi}=0,0170 ; \mathrm{p}>0,05)$; dizer aos irmãos (Phi $=0,033 ; \mathrm{p}>0,05)$; e dizer a um funcionário $(\mathrm{Phi}=0,062 ; \mathrm{p}>0,05)$.

No que se refere às reações dos alunos, de ambos os subgrupos investigados, de prestar auxílio quando vêem alguém fazendo mal a um estudante de mesma idade que a sua, constatou-se os seguintes comportamentos: tentar ajudar como pode $(n=167 ; 49,3 \%)$, ajudar mesmo que não conheça a pessoa $(n=87 ; 25,7 \%)$, chamar alguém para ajudar $(\mathrm{n}=78 ; 23 \%)$, não fazer nada para auxiliar (n $=58 ; 17,1 \%)$ e ajudar só se for amigo $(\mathrm{n}=50 ; 14,7 \%)$. Nesses itens, os estudantes também podiam assinalar mais de um comportamento. Destaca-se que os alunos com D\&T tenderam a apresentar os mesmos comportamentos que seus pares não identificados para lidarem com o bullying escolar: ajudar mesmo sem conhecer a pessoa $\left(\chi_{0}^{2}=1,343 ; \mathrm{gl}=1 ; \mathrm{p}>0,05\right)$, tentar ajudar como pode $\left(\chi_{0}^{2}=0,002 ; \mathrm{gl}=1 ; \mathrm{p}>0,05\right)$, ajudar apenas se for um amigo ou amiga $\left(\chi_{0}^{2}=0,604 ; \mathrm{gl}=1 ; \mathrm{p}>0,05\right)$ e, inclusive, não fazer nada em relação às práticas de agressões $\left(\chi_{0}^{2}=0,019 ; \mathrm{gl}=1 ; \mathrm{p}>0,05\right)$. No entanto, esses estudantes se diferenciaram significativamente de seus pares no comportamento referente a chamar outras pessoas para ajudarem $\left(\chi_{0}^{2}=5,691 ; \mathrm{gl}=1 ; \mathrm{p}<0,02\right)$, sendo que os estudantes com D\&T $(\mathrm{n}=21 ; 35,6 \%)$ adotam esse comportamento mais frequentemente se comparado ao grupo de alunos não identificados $(\mathrm{n}=57 ; 21 \%)$.

Ressalta-se que, no processo de identificação de talento, os alunos com D\&T foram classificados em um ou mais dos seguintes domínios: Capacidade Intelectual Geral ( $\mathrm{n}=39 ; 61,1 \%)$, Aptidão Acadêmica Específica (n $=32 ; 54,2 \%)$, Pensamento Criativo-Produtivo $(\mathrm{n}=22$; $37,3 \%)$, Capacidade de Liderança $(\mathrm{n}=7 ; 11,9 \%)$, Talento Especial para Artes $(\mathrm{n}=19 ; 32,2 \%)$, Capacidade Psicomotora $(n=5 ; 8,5 \%)$ e Capacidade Socioemocional $(\mathrm{n}=6 ; 10,2 \%)$. Essas informações foram obtidas através do contato com os profissionais participantes do projeto de identificação e desenvolvimento de estudantes talentosos implantado na instituição pesquisada. A Tabela 3 descreve a comparação entre envolvimento com o bullying e os domínios de talentos em que os alunos são identificados. Somente no domínio "Talento Especial para Artes" houve diferença significativa $(\mathrm{Phi}=0,369 ; \mathrm{p}<$ $0,05)$. Os alunos identificados com esse talento mostraram maior envolvimento com bullying como vítimas (70\%) e vítimas agressivas (80\%), se comparados a estudantes que foram identificados em outros domínios.

Tabela 3

Envolvimento com o Bullying de acordo com os Domínios de Talento

\begin{tabular}{|c|c|c|c|c|c|c|c|c|c|c|}
\hline \multirow{3}{*}{ Domínios } & \multirow{3}{*}{$\mathrm{D} \& \mathrm{~T}$} & \multicolumn{8}{|c|}{ Envolvimento com o bullying } & \multirow{3}{*}{$\begin{array}{c}\text { Comparações } \\
\text { intergrupos }\end{array}$} \\
\hline & & \multicolumn{2}{|c|}{$\begin{array}{c}\text { Não } \\
\text { envolvido }\end{array}$} & \multicolumn{2}{|c|}{ Vítima } & \multicolumn{2}{|c|}{ Agressor } & \multicolumn{2}{|c|}{$\begin{array}{l}\text { Vítima } \\
\text { Agressiva }\end{array}$} & \\
\hline & & $\mathrm{n}$ & $\%$ & $\mathrm{n}$ & $\%$ & $\mathrm{n}$ & $\%$ & $\mathrm{n}$ & $\%$ & \\
\hline Capacidade & Sim & 28 & 68,3 & 5 & 50,0 & 2 & 66,7 & 4 & 80,0 & Phi $=0,169 ; p>0,05$ \\
\hline Intelectual Geral & Não & 13 & 31,7 & 5 & 50,0 & 1 & 33,3 & 1 & 20,0 & \\
\hline Aptidão Acadêmica & Sim & 24 & 58,5 & 4 & 40,0 & 2 & 66,7 & 2 & 40,0 & Phi $=0,171 ; p>0,05$ \\
\hline Específica & Não & 17 & 41,5 & 6 & 60,0 & 1 & 33,3 & 3 & 60,0 & \\
\hline Pensamento & Sim & 16 & 39,0 & 4 & 40,0 & 1 & 33,3 & 1 & 20,0 & Phi $=0,112 ; p>0,05$ \\
\hline Criativo Produtivo & Não & 25 & 61,0 & 6 & 60,0 & 2 & 66,7 & 4 & 80,0 & \\
\hline \multirow{2}{*}{$\begin{array}{l}\text { Capacidade } \\
\text { de Liderança }\end{array}$} & Sim & 5 & 12,2 & 1 & 10,0 & 0 & 0 & 1 & 20,0 & Phi $=0,113 ; p>0,05$ \\
\hline & Não & 36 & 87,8 & 9 & 90,0 & 3 & 100 & 4 & 80,0 & \\
\hline \multirow{2}{*}{$\begin{array}{l}\text { Talento Especial } \\
\text { para Artes }\end{array}$} & Sim & 10 & 24,4 & 7 & 70,0 & 1 & 33,3 & 1 & 20,0 & $\mathrm{Phi}=0,369 ; \mathrm{p}<0,05$ \\
\hline & Não & 31 & 75,6 & 3 & 30,0 & 2 & 66,7 & 4 & 80,0 & \\
\hline Capacidade & Sim & 4 & 9,8 & 0 &, 0 & 1 & 33,3 & 0 & 0 & Phi $=0,256 ; p>0,05$ \\
\hline Psicomotora & Não & 37 & 90,2 & 10 & 100,0 & 2 & 66,7 & 5 & 100 & \\
\hline Talento & Sim & 4 & 9,8 & 1 & 10,0 & 0 & 0 & 1 & 20,0 & Phi $=0,122 ; p>0,05$ \\
\hline Socioemocional & Não & 37 & 90,2 & 9 & 90,0 & 3 & 100 & 4 & 80,0 & \\
\hline
\end{tabular}


Oliveira, J. C. \& Barbosa, A. J. G. (2012). Bullying entre Estudantes Com e Sem Características de Dotação e Talento.

\section{Discussão}

Quanto às características demográficas dos dois subgrupos comparados, as diferenças nas variáveis idade e ano escolar, isto é, os alunos com D\&T sendo mais jovens e de séries iniciais, podem ser decorrentes, dentre outros fatores, da especificidade da amostra. Reitera-se que o processo amostral não foi probabilístico, sendo os estudantes provenientes de uma única instituição.

A partir dos resultados obtidos, é possível observar que não houve diferenças entre os subgrupos participantes no que se refere ao envolvimento com bullying. Tanto os alunos com D\&T quanto os que não possuem tal característica demonstraram envolver-se de forma equivalente, seja como vítimas, agressores ou vítimas-agressivas. Ao analisar a prevalência encontrada do envolvimento com essa forma de violência (aproximadamente $30 \%$ para os dois subgrupos), notam-se semelhanças com os resultados de outras pesquisas em âmbito nacional (CEATS, 2010; Fante, 2005; Lopes Neto \& Saavedra, 2003).

Quanto à manifestação do bullying entre os alunos que não foram identificados com D\&T, percebe-se consonância com a literatura no que se refere ao predomínio de agressões não físicas (Pereira, 2008), à menor faixa etária e escolarização dos envolvidos (Lopes Neto, 2005) e à relação entre sexo e essa forma de agressão (Catini, 2004; Pereira, 2008; Smith, 2002), indicando que os meninos tendem a se envolver mais em situações de agressões físicas e as meninas demonstram maior envolvimento com as agressões não físicas. No entanto, no caso dos estudantes com D\&T essas diferenças não foram percebidas. Sugere-se, a partir desse resultado, que investigações sejam realizadas com amostras de discentes com D\&T mais amplas e obtidas de forma probabilística.

Os comportamentos dos alunos, de um modo geral, de tentar ajudar os pares que sofrem bullying podem contribuir para que a escola seja um ambiente de cooperação e de amizade. O resultado que evidenciou que os alunos com D\&T apresentam comportamento de auxílio às vítimas mais freqüentemente que os colegas coaduna com os estudos de Jackson, Moyle e Piechowski (2009) e Webb (1993). Esses autores afirmam que estudantes com D\&T tendem a apresentar níveis mais elevados de sensibilidade emocional e empatia. Não obstante, independentemente de acontecerem com maior ou menor frequência em indivíduos com D\&T, comportamentos prósociais, que vão contra as práticas violentas do bullying, devem ser constantemente valorizados e reforçados na instituição de ensino para que a escola possa ser um local que, além de promover aquisição de conhecimentos, também possa gerar um ambiente saudável, de segurança e de desenvolvimento social.

Os resultados do presente estudo, principalmente no âmbito escolar, devem nortear os esforços para minimizar as ocorrências de bullying. Uma estratégia que se destaca no combate a esse tipo de violência se refere a incentivar que os alunos relatem as situações de vitimização que vivenciem ou observem, uma vez que, apesar dos comportamentos de alguns estudantes de contar a alguém sobre as agressões que vêm sofrendo, ainda é comum que os mesmos mantenham-se calados. Os profissionais de ensino e familiares precisam estar cientes de que um aluno esteja sendo vítima de bullying. Incentivá-lo a contar faz com que seja possível a tomada de atitudes que possam coibir os maus tratos aos quais o aluno está exposto.

No que se refere aos domínios em que os participantes com D\&T foram identificados, os resultados revelaram que os alunos que possuem talento especial para artes tendem a ser mais vitimizados que os pares identificados em outros domínios. Essa constatação não encontra respaldo na literatura, seja confirmando-a ou refutando-a. As pesquisas da área sugerem, apenas, maior predisposição para problemas socioemocionais entre jovens com D\&T intelectual extremos (Alencar \& Virgolim, 2001; Peterson, 2009), mas sem especificarem, necessariamente, envolvimento desses indivíduos como vítimas do bullying. Ressalta-se que o programa de identificação de talentos ao qual esses participantes estão vinculados não discrimina níveis de desempenho intelectual, isto é, apenas classifica-os como com ou sem essa característica. Assevera-se, também, que são necessárias investigações sobre a vulnerabilidade e fatores de risco dos alunos com talento especial para artes, além de iniciativas que possam auxiliá-los na aquisição de estratégias de enfrentamento frente às práticas de bullying.

$O$ fato de os resultados mostrarem que alunos com D\&T não diferem significativamente dos pares sem $D \& T$ no que se refere à quantidade de amigos que possuem, bem como ao envolvimento com bullying, pode ser considerado um indicador de que esses estudantes possuem um nível de habilidades socioemocionais pelo menos equivalente ao dos alunos não identificados com $\mathrm{D} \& \mathrm{~T}$. Isso tem sido demonstrado na literatura sobre ajustamento socioemocional desses indivíduos (Lehman \& Erdwins, 2004; Luftig \& Nichols, 1990). Porém essas inferências devem ser alvo de estudos adicionais, já que não foram contempladas outras dimensões do desenvolvimento socioemocional (personalidade, habilidades sociais, etc.) dos estudantes.

As características metodológicas do estudo limitam a validade interna e externa desta pesquisa. Algumas delas, como o tipo e o tamanho da amostra, já foram direta ou indiretamente abordadas. Menciona-se, ademais, o fato de ter sido empregada somente uma medida de bullying baseada em autorrelato e aplicada em um único momento, sem, consequentemente, evidenciar-se de fato os perfis de vitimização e/ou agressão. Todavia, há que se destacar que a pesquisa aqui descrita possui validade ecológica, pelo menos, satisfatória. Desse modo, além de pesquisas longitudinais, com múltiplas medidas e que contem com amostras mais amplas e probabilísticas, re- 
comenda-se, também, associar bullying e D\&T a outras variáveis, tais como capacidades extremas - especialmente a intelectual -, características de personalidade e habilidades sociais.

Não obstante as limitações desta investigação, foi possível perceber que o bullying está presente na escola e afeta igualmente estudantes com e sem D\&T. Trata-se de um tema que, apesar de recente, desperta o interesse de educadores e pesquisadores devido ao caráter deletério que tem para os envolvidos, mas possui uma produção científica muito limitada no Brasil.

O fato de os alunos com e sem D\&T não diferirem em termos de envolvimento com o bullying permite propor intervenções sociais iguais para ambos os grupos. Se não houver tentativas de combater essa forma de violência, as consequências do descaso podem ser extremamente nocivas, porque não só os envolvidos diretamente como também a comunidade escolar e a sociedade estarão expostos aos efeitos prejudiciais do bullying. Assim, faz-se necessária uma atenção especial por parte da família, da escola, da comunidade e das pessoas que formulam políticas públicas, no sentido de estarem alertas para os casos de bullying, identificando-os e intervindo. Combater não basta. Há que se elaborarem e implementarem projetos de prevenção para todos os alunos, com e sem D\&T.

\section{Referências}

Alencar, E. M. L. S. (2007). Características sócio-emocionais do superdotado: Questões atuais. Psicologia em Estudo, 12(2), 371-378.

Alencar, E. M. L. S., \& Fleith, D. S. (2001). Superdotados: Determinantes, educação e ajustamento. São Paulo, SP: Editora Pedagógica e Universitária.

Alencar, E. M. L., \& Virgolim, A. M. R. (2001). Dificuldades emocionais e sociais do superdotado. In E. M. L. S. Alencar (Ed.), Criatividade e educação dos superdotados (pp. 174205). Petrópolis, RJ: Vozes.

Almeida, K. L., Silva, A. C., \& Campos, J. S. (2008). Importância da identificação precoce da ocorrência do bullying: Uma revisão de literatura. Revista de Pediatria, 9(1), 8-16.

Associação Brasileira de Empresas de Pesquisa. (2010). Critério Brasil de Classificação Econômica. Retrieved October 24, 2010, from http://www.abep.org/novo/

Barbosa, A. J. G. (2009). Questionário de Bullying. Juiz de Fora, MG: Autor.

Barbosa, A. J. G., Ghenther, Z. C., Pereira, C. E. S., Gonçalves, F. C., Oliveira, E. A., Scoralick, N. N., et al. (2008). Altas habilidades/superdotação: Estratégias de identificação e alternativas educacionais para estudantes talentos. In A. J. B. Barbosa, L. M. Lourenço, \& M. M. P. E. Mota (Eds.), Desenvolvimento psicossocial: Temas em educação e saúde (pp. 33-51). Campinas, SP: Alínea.

Botelho, R. G., \& Souza, J. M. C. (2007). Bullying e educação física na escola: Características, casos, conseqüências e estratégias de intervenção. Revista de Educação Física, 139, 58-70.

Carter, B. B., \& Spencer, V. G. (2006). The fear factor: Bullying and students with disabilities. International Journal of Special Education, 21(1), 1-14.
Catini, N. (2004). Problematizando o "bullying" para a realidade brasileira. (Tese de Doutorado não-publicada). Programa de Pós-Graduação em Psicologia, Centro de Ciências da Vida, Pontifícia Universidade Católica de Campinas, SP.

Centro de Empreendedorismo Social e Administração em Terceiro Setor. (2010). Bullying escolar no Brasil. São Paulo, SP: Autor

Chagas, J. F. (2008). Adolescentes talentosos: Características individuais e familiares. (Tese de Doutorado não-publicada). Instituto de Psicologia, Universidade Federal de Brasília, DF.

Cross, T. L. (2001). Social/Emotional needs: The rage of gifted students. Gifted Child Today, 24(2), 43-45.

Estatuto da Criança e do Adolescente. (1990). Lei n. 8.069, de 13 de julho de 1990, Lei n. 8.242, de 12 de outubro de 1991 (3. ed.). Brasília, DF: Câmara dos Deputados.

Estell, D. B., Farmer, W. F., Irvin, M. J., Crowther, A., Akos, P., \& Boudah, D. J. (2009). Students with exceptionalities and the peer group context of bullying and victimization in late elementary. Journal of Child and Family Studies, 18, 136-150.

Fante, C. (2005). Fenômeno Bullying: Como prevenir a violência nas escolas e educar para a paz. Campinas, SP: Verus.

Flynt, S. W., \& Morton, R. C. (2007). Bullying prevention and students with disabilities. National Forum of Special Education Journal, 19(1), 1-6.

Güenther, Z. C. (2006). Desenvolver capacidades e talentos: Um conceito de inclusão (2. ed.). Petrópolis, RJ: Vozes.

Hay, P. (2008). Prosocial reasoning and empathy in gifted children. (Unpublished doctoral dissertation). Department of Philosophy, University of New South Wales, Sydney, Australia.

Horowitz, F. D. (2009). A developmental understanding of giftedness and talent. In F. D. Horowitz, R. F. Subotnik, \& D. J. Matthews (Eds.), The development of giftedness and talent across the life span (pp. 3-20). Washington, DC: American Psychological Association.

Instituto Brasileiro de Geografia e Estatística. (2003). Metodologia do Censo Demográfico 2000: Vol. 25. Rio de Janeiro, RJ: Autor.

Jackson, P. S., Moyle, V. F., \& Piechowski, M. M. (2009). Emotional life and psychotherapy of the gifted in light of Dabrowski's theory. In L. V. Shavinina (Ed.), International handbook on giftedness (pp. 437-465). New York: Springer.

Kaufman, S. B., \& Sternberg, R. J. (2008). Conceptions of giftedness. In S. Pfeiffer (Ed.), Handbook of giftedness in children: Psycho-educational theory, research, and best practices. Tallahassee, FL: Springer.

Lehman, E. B., \& Erdwins, C. J. (2004). The social and emotional adjustment of young, intellectually gifted children. In S. M. Moon (Ed.), Social/emotional issues, underachievement, and counseling of gifted and talented students (pp. 1-8). Thousand Oaks, CA: Corwin Press.

Lisboa, C., Braga, L. L., \& Ebert, G. (2009). O fenômeno bullying ou vitimização entre pares na atualidade: Definições, formas de manifestação e possibilidades de intervenção. Contextos Clínicos, 2(1), 59-71.

Lopes Neto, A. A. (2005). Bullying - Comportamento agressivo entre estudantes. Jornal de Pediatria, 81(5), 164-172.

Lopes Neto, A. A., \& Saavedra, L. H. (2003). Diga não ao Bullying: Programa de redução do comportamento agressivo entre estudantes - Resultados parciais. Retrieved April 15, 2010, from http://www.observatoriodainfancia.com.br 
Oliveira, J. C. \& Barbosa, A. J. G. (2012). Bullying entre Estudantes Com e Sem Características de Dotação e Talento.

Luftig, R. L., \& Nichols, M. L. (1990). Assessing the social status of gifted students by their age peers. Gifted Child Quarterly, 34, 111-115.

Malta, D. C., Silva, M. A. I., Mello, F. C. M., Monteiro, R. A., Sardinha, L. M. V., Crespo, C., et al. (2010). Bullying nas escolas brasileiras: Resultados da Pesquisa Nacional de Saúde do Escolar (PeNSE), 2009. Ciência \& Saúde Coletiva, 15(Supl. 2), 3065-3076.

Ministério da Educação. (2008). Política Nacional de Educação Especial na Perspectiva da Educação Inclusiva. Documento elaborado pelo Grupo de Trabalho nomeado pela Portaria ${ }^{\circ}$ $555 / 2007$, prorrogada pela Portaria $n^{\circ} 948 / 2007$, entregue ao Ministro da Educação em 07 de janeiro de 2008. Retrieved April 15, 2010, from http://portaldoprofessor. mec.gov.br/ storage/materiais/0000011730.pdf

Nansel, T. R., Craig, W., Overpeck, M. D., Saluja, G., \& Ruan, W. J. (2004). Cross-national consistency in the relationship between bullying behaviors and psychosocial adjustment. Archives of Pediatrics \& Adolescent Medicine, 158(8), 730-736.

Nogueira, R. M. C. P. A. (2005). A prática de violência entre pares: O bullying nas escolas. Revista Iberoamericana de Educación, 37, 93-102.

Oliveira, F. F., \& Votre, S. J. (2006). Bullying nas aulas de educação física. Revista Movimento, 12(2), 173-197.

Oliveira, J. M. (2007). Indícios de casos de bullying no ensino médio de Araraquara - SP. (Dissertação de Mestrado nãopublicada). Centro Universitário de Araraquara, Universidade de Araraquara, SP.

Olweus, D. (1993). Bullying at school: What we know and what we can do. Oxford, UK: Blackwell.

Ourofino, V. T. A. T., \& Guimarães, T. G. (2007). Características intelectuais, emocionais e sociais do aluno com altas habilidades/superdotação. In D. Fleith (Ed.), A construção de práticas educacionais para alunos com altas habilidades/ superdotação: Vol. 1 (pp. 41-52). Brasília, DF: Ministério da Educação.

Parker, M. R. (2010). A comparison of bullying and victimization rates among gifted and High-Achieving students. (Unpublished doctoral dissertation). Department of Philosophy, University of Tennessee, Knoxville, TN.

Pereira, B. O. (2008). Para uma escola sem violência: Estudo e prevenção das práticas agressivas entre crianças (2. ed.). Lisboa, Portugal: Ministério da Ciência e da Tecnologia.

Pereira, C. E. S. (2010). Identificação de estudantes talentosos: Uma comparação entre as perspectivas de Renzulli e Güenther. (Dissertação de Mestrado não-publicada). Programa de Pós-Graduação em Psicologia, Universidade Federal de Juiz de Fora, MG.

Peterson, J. S. (2001). Gifted and at risk: Four longitudinal case studies of post-high-school development. Roeper Review, 24, 31-39.

Peterson, J. S. (2009). Myth 17: Gifted and talented individuals do not have unique social and emotional needs. Gifted Child Quarterly, 53(4), 280-282.

Peterson, J. S., \& Ray, K. E. (2006a). Bullying and the gifted: Victims, perpetrators, prevalence, and effects. Gifted Child Quaterly, 50(2), 148-168.

Peterson, J. S., \& Ray, K. E. (2006b). Bullying among the gifted: The subjective experience. Gifted Child Quaterly, 50(3), 252269.
Piedra, R. R., Lago, A. S., \& Massad, J. L. P. (2006). Crianças contra crianças: O bullying, uma perturbação emergente. Anales de Pediatría, 1(2), 101-104.

Preuss, L. J., \& Dubow, E. F. (2004). A comparison between intellectually gifted and typical children in their coping responses to a school and a peer stressor. Roeper Review, 26, 105-111.

Schuler, P. A. (2002). Teasing and gifted children. The SENG Newsletter, 2(1), 3-4.

Secretaria de Educação Especial. (1995). Subsídios para a organização e funcionamento de serviços em educação especial: Área de altas habilidades. Brasília, DF: Ministério da Educação.

Seixas, S. R. (2005). Violência escolar: Metodologias de identificação dos alunos agressores e/ou vítimas. Análise Psicológica, 2(23), 97-110.

Smith, P. K. (2002). Intimidação por colegas e maneiras de evitá-la. In E. Debardieux \& C. Blaya (Eds.), Violência nas escolas e politicas públicas (pp. 187-205). Brasília, DF: Organização das Nações Unidas para a Educação, a Ciência e a Cultura.

Webb, J. T. (1993). Nurturing social-emotional development of gifted children. In K. A. Heller, F. J. Mönks, \& A. H. Passow (Eds.), International handbook of research and development of giftedness and talent (pp. 525-538). Oxford, UK: Pergamon. 